Rev. Int. Contam. Ambie. 37, 293-306, 2021

https://doi.org/10.20937/RICA.53860

\title{
BIOMONITOREO ACTIVO CON Leskea angustata EN LA ZONA METROPOLITANA DEL VALLE DE TOLUCA
}

Active biomonitoring with Leskea angustata in the metropolitan zone of the Toluca valley

\author{
Nancy LARA-ALMAZÁN ${ }^{1,2}$, Graciela ZARAZÚA-ORTEGA ${ }^{2 *}$, \\ María de los Ángeles GARCÍA-CHÁVEZ ${ }^{1}$, Ana Marcela GÓMEZ-HINOJOS ${ }^{3}$, \\ Carlos Eduardo BARRERA-DÍAZ ${ }^{4}$ y Pedro ÁVILA-PÉREZ ${ }^{5}$
}

${ }^{1}$ Facultad de Química, Universidad Autónoma del Estado de México, Paseo Colón s/n, Residencial Colón, 50120 Toluca, Estado de México, México.

${ }^{2}$ Laboratorio Nacional de Investigaciones en Forense Nuclear, Instituto Nacional de Investigaciones Nucleares, carretera México-Toluca s/n, La Marquesa, 52750 Ocoyoacac, Estado de México, México.

${ }^{3}$ Facultad de Planeación Urbana y Regional, Universidad Autónoma del Estado de México, Paseo Colón s/n, Residencial Colón, 50120 Toluca, Estado de México, México.

${ }^{4}$ Secretaría de Investigación y Estudios Avanzados, Universidad Autónoma del Estado de México, Paseo Colón s/n, Residencial Colón, 50120 Toluca, Estado de México, México.

${ }^{5}$ División de Estudios de Posgrado e Investigación, Instituto Tecnológico de Toluca, Apartado Postal 890, 52140 Metepec, México, México.

*Autora para correspondencia: graciela.zarazua@inin.gob.mx

(Recibido: enero de 2020; aceptado: mayo de 2020)

Palabras clave: metales pesados, bolsas de musgo, fitoquelatinas, TXRF, UPLC-MS

\section{RESUMEN}

El objetivo de este trabajo fue evaluar los niveles de $\mathrm{Cu}, \mathrm{Zn}$ y $\mathrm{Pb}$ en la zona metropolitana del valle de Toluca (ZMVT) empleando la técnica de biomonitoreo activo con bolsas con musgo (moss bags) con Leskea angustata como bioindicador, así como determinar si se producen fitoquelatinas (PC) y si hay correlación entre la concentración de éstas y de glutatión (GHS) con los metales presentes en el musgo. Se seleccionaron 10 sitios de monitoreo y en cada uno se colocaron dos bolsas con musgo para ser expuestas al ambiente de la ZMVT durante seis meses. Las concentraciones de metales se determinaron mediante la técnica de fluorescencia de rayos x en reflexión total (TXRF), mientras que el GHS y las PC se cuantificaron empleando la técnica de cromatografía de líquidos de ultra alta resolución acoplada a espectrometría de masas (UPLC-MS). Se evaluó la concentración de metales, PC y GHS antes y después de la exposición y se calculó la tasa de enriquecimiento. Los resultados muestran enriquecimientos de 2 a 30 para metales y de 116 a 569 para GHS. La concentración de PC siempre fue menor al límite de detección. El orden de la concentración total de metales en los sitios de monitoreo fue: Tollocan $>$ Reforma $>$ San Mateo Atenco $>$ Alameda $>$ Aeropuerto $>$ San Cristóbal $>$ Metepec $>$ Ceboruco $>$ Toluca Centro $>$ Oxtotitlán. En general Tollocan, San Mateo Atenco, Alameda y Reforma fueros significativamente diferentes a los demás sitios. El GHS presentó un rango de concentración de 164 a $808 \mathrm{nM} / \mathrm{g}$ y presentó correlaciones positivas $(\mathrm{r}=0.8)$ con el $\mathrm{Cu}, \mathrm{Zn}$ y $\mathrm{Pb}$, por lo que se infiere que la concentración de los metales en estudio tiene relación directa con su inducción. Con los resultados obtenidos en este trabajo se pude concluir que el GHS desempeña un papel fundamental en la bioacumulación de metales divalentes en Leskea angustata de la ZMVT e incrementa su potencial de acumulación, ya que es un mecanismo importante de destoxificación en estos organismos. 
Key words: heavy metals, moss bags, phytochelatins, TXRF, UPLC-MS

\begin{abstract}
The main purpose of this research was to assess $\mathrm{Cu}, \mathrm{Zn}$, and $\mathrm{Pb}$ levels in the metropolitan area of the Toluca Valley (ZMVT, Spanish acronym) by active biomonitoring, using moss bags and Leskea angustata as bioindicators, as well as to determine whether phytoquelatins (PCs) are produced and if there is a correlation between PCs and glutathione (GHS) concentrations and the heavy metals present in moss. Ten monitoring locations were selected in which two moss bags were exposed to the ZMVT environment for six months. Heavy metals concentrations were determined using total reflection X-ray fluorescence (TRXF), whereas GHS and PCs were quantified by ultra-performance liquid chromatography-tandem mass spectrometer (UPLC-MS). GHS and PCs concentration were measured before and after exposition, and the enrichment rate was also calculated. Results show enrichment rates between 2 and 30 for heavy metals and 116 and 569 for GHS. PCs concentrations were below the limit of detection. The order of total metal concentrations in the monitoring locations was: Tollocan $>$ Reforma $>$ San Mateo Atenco $>$ Alameda $>$ Aeropuerto $>$ San Cristóbal $>$ Metepec $>$ Ceboruco $>$ Toluca Centro > Oxtotitlán. Overall, Tollocan, San Mateo Atenco, Alameda and Reforma were significantly different from other locations. GHS presented a concentration rate between 164 and $808 \mathrm{nM} / \mathrm{g}$ and had positive correlations $(\mathrm{r}=0-8)$ with $\mathrm{Cu}, \mathrm{Zn}$ and $\mathrm{Pb}$; therefore, it is inferred that concentrations of the assessed heavy metals in this study are related to their direct induction. It can be concluded that GHS plays a major role in divalent metals bioaccumulation since it is an important detoxification mechanism in these organisms.
\end{abstract}

\section{INTRODUCCIÓN}

Debido a la relación que existe entre la contaminación atmosférica, los efectos en la salud y los costos asociados, el monitoreo de la calidad del aire ha cobrado en la actualidad una importancia fundamental para identificar y proveer información necesaria acerca de las concentraciones de contaminantes en el aire de cada región y sus tendencias. En México existen redes automáticas de monitoreo atmosférico (RAMA) encargadas de monitorear los contaminantes criterio (partículas suspendidas totales [PST], partículas menores a 10 micrómetros $\left[\mathrm{PM}_{10}\right]$, partículas menores a 2.5 micrómetros $\left[\mathrm{PM}_{2.5}\right]$, ozono $\left[\mathrm{O}_{3}\right]$, dióxido de nitrógeno $\left[\mathrm{NO}_{2}\right]$, dióxido de azufre $\left[\mathrm{SO}_{2}\right]$ y monóxido de carbono [CO]), así como parámetros meteorológicos. En la zona metropolitana del valle de Toluca (ZMVT) se ubica una RAMA que comenzó a operar en 1993. A partir de esa fecha ha sido el sistema que proporciona información sobre la calidad del aire de esta zona (SEMARNAT 2012, GEM 2014).

La contaminación atmosférica, se puede definir como la presencia de uno o más elementos (polvo, olores, humos, vapor, agentes químicos) con ciertas características, en cantidad suficiente y con una permanencia determinada, que puedan causar efectos indeseables tanto al ser humano como a vegetación, animales, construcciones y monumentos. En la ZMVT, el crecimiento poblacional, urbano e industrial ha generado un aumento en la demanda de servicios públicos, transporte y vivienda. Como resultado de éstas y otras actividades se emiten anualmente más de 600 mil toneladas de contaminantes a la atmósfera. El parque vehicular contribuye con aproximadamente 565 mil toneladas de contaminantes al año; los procesos de transformación industrial, comercial y de servicios contribuyen con 36 mil toneladas, y los procesos activos de extracción de arena, grava y tepetate con 2205 toneladas. Aunado a lo anterior, es muy probable que no exista una dispersión adecuada de contaminantes (incluyendo el material particulado [MP]), debido a las condiciones atmosféricas que se presentan en el área, el medio físico que la rodea y las actividades antrópicas que en ella se realizan (SMA 2012).

El origen del MP puede ser tanto natural (erupciones volcánicas, tolvaneras e incendios forestales) como antrópico (principalmente de la quema de combustibles fósiles). Dentro de su composición se encuentran los metales pesados, que representan un problema ya que no pueden degradarse biológicamente, por lo que persisten en el ambiente. Además, el MP puede depositarse en áreas lejanas a su fuente de 
origen a causa de la circulación de los vientos, (Keshavarzi et al. 2015). La Organización Mundial de la Salud (OMS) señala que el MP es un contaminante perjudicial para la salud y el bienestar de los seres humanos. La exposición a estos contaminantes se ha relacionado con trastornos pulmonares y enfermedades cardiovasculares (OMS 2016).

Aunque la ZMVT cuenta con una RAMA, ésta únicamente reporta datos sobre contaminantes criterio. No contempla la concentración de metales, por lo que una alternativa para evaluar la presencia e impacto de metales es el uso de especies vegetales como bioindicadores de contaminación atmosférica. En los últimos años, el biomonitoreo se ha convertido en una alternativa a los métodos convencionales. Con este fin se han utilizado hojas y cortezas de árbol, líquenes y musgos, entre otros (Cocozza et al. 2016, Gómez et al. 2013, Špirić et al. 2013).

Las briofitas, el segundo grupo más importante de plantas verdes, se dividen en tres categorías: antocerotes, hepáticas y musgos. Generalmente son pequeñas y habitan en ambientes muy variados, desde el nivel del mar hasta alturas superiores a los $3000 \mathrm{~m}$ sobre el nivel del mar. La mayoría de sus familias son cosmopolitas, aunque altamente dependientes de la humedad ambiente (Zechmeister et al. 2003). Se ha demostrado que por sus características morfológicas, fisiológicas y geográficas, los musgos son organismos idóneos para el biomonitoreo atmosférico, ya que no tienen raíces reales y obtienen los nutrientes directamente de la atmósfera. La conducción externa del agua y nutrientes mediante capilaridad a lo largo de la superficie de la planta es la que resulta fisiológicamente más significativa y fundamental para conservar y acumular los elementos que llegan pasivamente a través de la sedimentación seca y húmeda del ambiente (Basile et al. 2012, Bačeva et al. 2013, Gerdol et al. 2014).

El biomonitoreo puede ser pasivo o activo. En el primer caso, los bioindicadores son recolectados directamente del sitio de estudio, mientras que, en el segundo, los organismos biológicos son transferidos de un cultivo in vitro o de un hábitat relativamente libre de contaminación a los sitios en que se desea monitorear la contaminación del aire (Ares et al. 2012). En este ultimo caso, la técnica de bolsas con musgo es la más utilizada y se ha aplicado sobre todo en estudios urbanos, donde la presencia de musgo es poca o nula. El biomonitoreo activo tiene la ventaja de que es posible calcular la tasa de enriquecimiento de los contaminantes, así como controlar el tiempo de exposición (Aničić et al. 2009, Tretiach et al. 2011, Giordano et al. 2009, Vuković et al. 2014, Calabrese et al. 2015, González et al. 2016).
En bajas concentraciones, metales como $\mathrm{Cu}$ y $\mathrm{Zn}$ son esenciales para las plantas, ya que se requieren para la biosíntesis de enzimas y para su crecimiento y metabolismo; sin embargo, en concentraciones mayores pueden causar daños, al igual que los considerados no esenciales como $\mathrm{Cd}, \mathrm{Hg}$ y $\mathrm{Pb}$. Cuando se incorporan metales tóxicos en una especie vegetal, para contrarrestar su exceso algunas de éstas han desarrollado mecanismos celulares que intervienen en la tolerancia a elementos tóxicos, entre los que se encuentran el glutatión (GSH, por sus siglas en inglés) y las fitoquelatinas (PC, por sus siglas en inglés), los cuales forman complejos con metales principalmente divalentes $(\mathrm{Cd}, \mathrm{Cu}, \mathrm{Hg}, \mathrm{Pb}, \mathrm{Zn})$ (Cobbett 2000, Bruns et al. 2001).

Las plantas que viven en comunidades naturales dependen de numerosos mecanismos de defensa para hacer frente al estrés abiótico. Los mecanismos que permiten a las plantas percibir las condiciones ambientales en las que crecen y responder a los factores estresantes abióticos consisten en cadenas complejas de reacciones múltiples. En los últimos años se ha investigado el metabolismo del azufre, en particular del GSH, que es un tripéptido formado por glutamato $(\boldsymbol{\gamma}$-Glu), cisteína (Cys) y glicina (Gly), ya que participa en muchos de los procesos metabólicos de una célula vegetal y es un componente esencial del sistema antioxidante que controla las especies reactivas de oxígeno y el grupo tiol (-SH) de la cisteína; está relacionado con la quelación de xenobióticos y metales pesados. A diferencia de las plantas superiores, en las briofitas el GSH parece desempeñar un papel fundamental en la desintoxicación de metales mediante el transporte de éstos a través del citoplasma (Bruns et al. 2001, Saxena et al. 2003, Bleuel et al. 2005, Braha et al. 2007, Rau et al. 2007, Leinenweber et al. 2009, Emamverdian et al. 2015).

Por otro lado, las PC son péptidos que se producen únicamente en presencia de metales pesados como $\mathrm{Cu}, \mathrm{Zn}$ y $\mathrm{Pb}$ a partir de péptidos de bajo peso molecular (entre 2 y $10 \mathrm{KDa}$ ) como el GSH y el aminoácido cisteína (Cys). El GSH es el sustrato crucial para la síntesis de PC (Steffens 1990, Grill et al. 1991, Kneer y Zenk 1992, Bruns et al. 2001). Las PC contribuyen a la depuración de metales, así como al mantenimiento del balance de metales esenciales en las células y al transporte de formas reducidas del azufre asimilado (Clemens y Peršoh 2009, Konno et al. 2010). La estructura de las PC es la siguiente: ( $\gamma$-Glu-Cys)n Gly, donde $\mathrm{n}=2-11$, siendo las más comunes las PC2, PC3, PC4 y PC5 (Cobbett 2000, Cobbett y Goldsbrough 2002). 
Se tienen antecedentes de PC en musgos. Gekeler et al. (1989) detectaron la presencia de PC2 y PC3 en Marchantia polymorpha; Bruns et al. (1997) reportaron PC con una alta correlación con Cd en Fontinalis antipyretica; Saxena et al. (2003) evaluaron el potencial de bioacumulación de $\mathrm{Pb}$ en el musgo Sphagnum squarrosum, y encontraron que dicho metal induce la síntesis de PC; Krauss et al. (2005) establecieron que la destoxificación de Cd en Marchantiophyta ocurre por la inducción de las PC2 y PC4; Petraglia et al (2014) reportaron la presencia de PC2, PC3, PC4 en Sphagnum palustre, y Gil (2015) encontró concentraciones de 23 a $39 \mathrm{mg} / \mathrm{kg}$ de PC4 en el musgo epífito Fabronia ciliaris de la ZMVT.

El objetivo de este trabajo fue evaluar los niveles de $\mathrm{Cu}, \mathrm{Zn}$ y $\mathrm{Pb}$ en la ZMVT empleando la técnica de biomonitoreo activo con bolsas con musgo con Leskea angustata como bioindicador, así como determinar si se producen PC y si hay correlación entre su concentración y la de GSH con los metales presentes en el musgo.

\section{MATERIALES Y MÉTODOS}

\section{Área de estudio}

La ZMVT tiene una superficie de $1208 \mathrm{~km}^{2}$ y se ubica en la zona centro del Estado de México, entre los paralelos $18^{\circ} 59^{\prime} 07^{\prime \prime}$ y $19^{\circ} 34^{\prime} 47^{\prime \prime} \mathrm{N}$ y los meridianos $99^{\circ} 38^{\prime} 22^{\prime \prime}$ y 99 56' 13" O (Fig. 1). Según la clasificación de Köppen, el clima de la ZMVT se cataloga como $\mathrm{C}(\mathrm{w} 2)(\mathrm{w}) \mathrm{bi}(\mathrm{g})$, que corresponde a un clima semifrío subhúmedo (el más húmedo en su categoría), con lluvias de verano y un porcentaje de lluvia invernal menor al $5 \%$. La temporada de lluvias comienza a mediados de mayo y termina a mediados de octubre. La precipitación media anual supera los $800 \mathrm{~mm}$ de lluvia. La temperatura en zonas con altitud mayor a los $3000 \mathrm{msnm}$ varía entre de -5 a $10{ }^{\circ} \mathrm{C}$, mientras que en el valle varía de 4 a $26^{\circ} \mathrm{C}$, presentándose las máximas temperaturas antes del solsticio de verano (Díaz et al. 2005, SMA 2012).

El relieve y la orografía, además del viento, son variables que desempeñan un papel importante en la dispersión y acumulación de contaminantes atmosféricos. La ZMVT se encuentra rodeada por un valle de origen tectónico con manifestaciones volcánicas recientes y procesos activos de erosión y sedimentación. Colinda al oeste con el sistema montañoso del Nevado de Toluca y Santa María del Monte, al sur con la sierra de Tenango, al este con el sistema de la sierra de las Cruces y Monte Alto, y al norte con la falla de Perales. Los vientos predominantes son alisios, los cuales corren a lo largo de la ZMVT de sureste a noroeste (Fig. 1) (SMA 2012, GEM 2014).

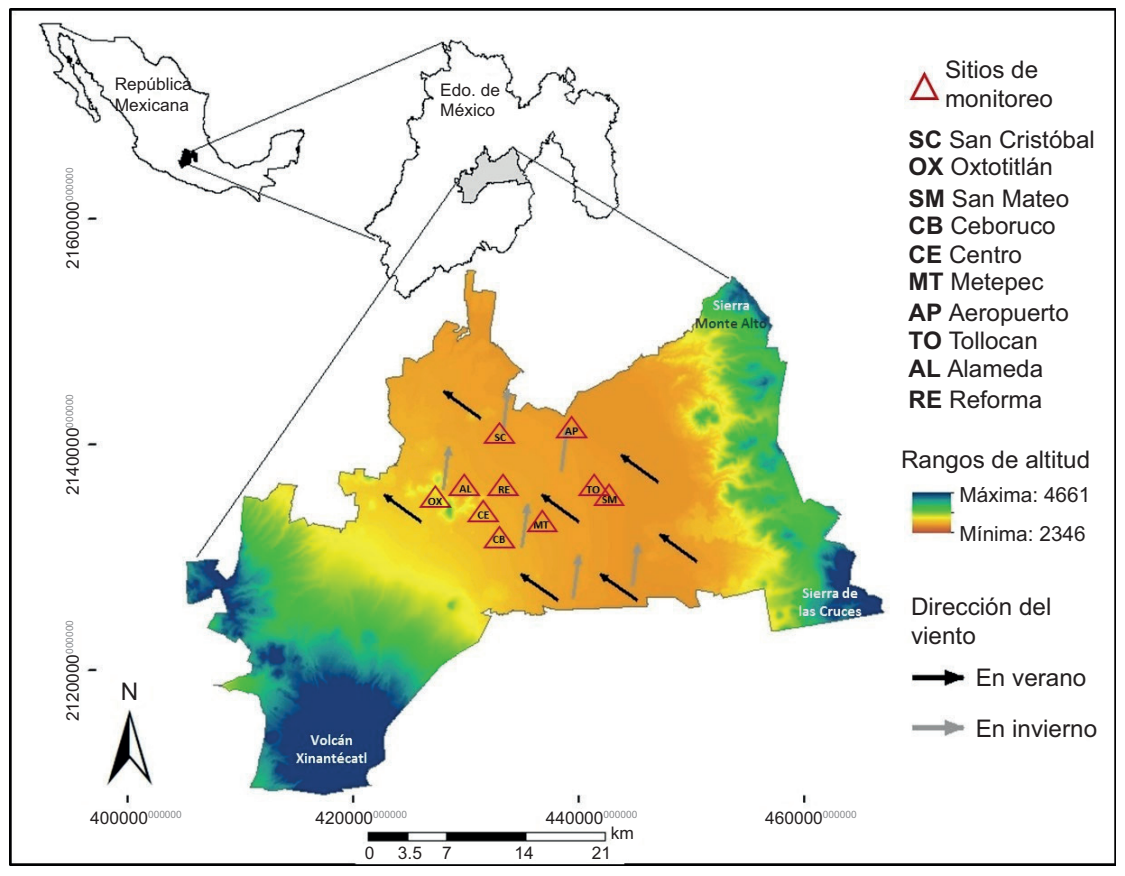

Fig. 1. Localización de la zona metropolitana del valle de Toluca y sitios de monitoreo. 
Para llevar a cabo la exposición de las bolsas con musgo se seleccionaron 10 sitios de monitoreo: siete correspondientes a las estaciones de la RAMA y tres más caracterizados por presentar concentraciones elevadas de metales (Cuadro I) (Zarazúa-Ortega et al. 2013).

\section{Selección del musgo}

En este estudio se consideró utilizar un musgo epífito que fuera una especie abundante en la ZMVT y un buen acumulador de metales. De acuerdo con Zepeda-Gómez et al. (2012), Leskea angustata es una de las especies de musgo más abundantes en la ZMVT, mientras que Poblano (2013) la reportó como hiperacumuladora de $\mathrm{Cu}, \mathrm{Zn}$ y $\mathrm{Pb}$ con factores de enriquecimiento $\geq 10$. El musgo L. angustata fue recolectado in situ dentro del Instituto Nacional de Investigaciones Nucleares (ININ) ubicado en Ocoyoacac, Estado de México, zona que ha sido reportada como poco impactada por metales de origen antrópico (Poblano 2013, Dotor 2014, Tejeda et al. 2015). El musgo completo (rizoides, gametofitos y esporofitos) fue tomado de la corteza de árboles a una altura de 2 a $3 \mathrm{~m}$; una vez en el laboratorio, se lavó suavemente con agua y se sometió a una selección más detallada con el fin de obtener únicamente la especie L. angustata. Posteriormente se colocó en charolas de polietileno y se hidrató durante una semana con el objeto de que se pudiera manipular al introducirlo en las bolsas.

Aunque se ha reportado que la exposición de musgo desvitalizado tiene numerosas ventajas (Ares et al. 2012), para este estudio se consideró utilizar monitoreo activo con musgo vivo con el objeto de comparar los resultados con trabajos previos realizados en la misma zona de estudio (Poblano 2013, Zarazúa-Ortega et al. 2013, Macedo-Miranda et al. 2016), en los cuales se aplicó monitoreo pasivo, y evaluar las ventajas de aplicar monitoreo activo con bolsas con musgo.

\section{Bolsas con musgo}

De acuerdo con la literatura revisada, la mayoría de los autores emplean malla plástica con una apertura de 4 a $5 \mathrm{~mm}$ para la elaboración de las bolsas con musgo. Las formas más utilizadas son la esférica y la rectangular (de $15 \times 5-10 \mathrm{~cm})$. Se recomienda colocar de dos a tres bolsas por sitio con 100 y $500 \mathrm{mg}$ (peso seco) de musgo, en función de la disponibilidad de éste, del tipo y cantidad de análisis y de la posible pérdida durante su exposición. El periodo de exposición varía de una semana a 20 meses, siendo el más común de tres meses, ya que en periodos mayores a dos meses se obtiene menos variabilidad. Esto se debe a que la acumulación de metales en los musgos no es lineal, aunque generalmente aumenta con el tiempo (Adamo et al. 2003, Tretiach et al. 2007, Giordano et al. 2009, Ares et al. 2012, Calabrese et al. 2015).

Tomando en cuenta estas consideraciones, las bolsas con musgo se elaboraron con tela plástica tipo malla de $5 \mathrm{~mm}$ de abertura de forma rectangular $(20 \times$ $15 \mathrm{~cm}$ ) dentro de las cuales se colocaron las muestras de musgo vivo (aproximadamente $20 \mathrm{~g}$ de musgo hidratado), cubriendo perfectamente el área de las bolsas, y éstas se fijaron en soportes de madera. En cada sitio de monitoreo se colocaron dos bolsas con musgo orientadas de sur a norte y se dejaron expues-

CUADRO I. SITIOS DE MONITOREO ACTIVO EN LA ZONA METROPOLITANA DEL VALLE DE TOLUCA.

\begin{tabular}{|c|c|c|c|}
\hline \multirow{2}{*}{ Sitios de monitoreo } & \multirow{2}{*}{ Zona } & \multicolumn{2}{|c|}{ Coordenadas } \\
\hline & & Latitud Norte & Longitud Oeste \\
\hline San Cristóbal (RAMA) & Norte & $19^{\circ} 19^{\prime} 38.0 "$ & $99^{\circ} 38^{\prime} 3.44^{\prime \prime}$ \\
\hline Aeropuerto (RAMA) & Norte & $19^{\circ} 20^{\prime} 4.41 ”$ & $99^{\circ} 34^{\prime} 26.3 ”$ \\
\hline Oxtotitlán (RAMA) & Centro & $19^{\circ} 17^{\prime} 0.40^{\prime \prime}$ & $99^{\circ} 41^{\prime} 0.56^{\prime \prime}$ \\
\hline Toluca Centro (RAMA) & Centro & $19^{\circ} 16^{\prime} 41.1 "$ & $99^{\circ} 39^{\prime} 23.1^{\prime \prime}$ \\
\hline Metepec (RAMA) & Sur & $19^{\circ} 16^{\prime} 12.7^{\prime \prime}$ & $99^{\circ} 35^{\prime} 42.7^{\prime \prime}$ \\
\hline Ceboruco (RAMA) & Sur & $19^{\circ} 15^{\prime} 37.1 ”$ & $99^{\circ} 38^{\prime} 44.6^{\prime \prime}$ \\
\hline San Mateo Atenco (RAMA) & Sur & $19^{\circ} 16^{\prime} 49.5^{\prime \prime}$ & $99^{\circ} 32^{\prime} 30.0^{\prime \prime}$ \\
\hline Tollocan & Sur & $19^{\circ} 17^{\prime} 25^{\prime \prime}$ & $99^{\circ} 39^{\prime} 41.6 \prime$ \\
\hline Alameda & Centro & $19^{\circ} 17^{\prime} 24^{\prime \prime}$ & $99^{\circ} 38^{\prime} 31.1 ”$ \\
\hline Reforma & Centro & $19^{\circ} 17^{\prime} 10.6^{\prime \prime}$ & $99^{\circ} 33^{\prime} 0.3^{\prime \prime}$ \\
\hline
\end{tabular}

RAMA: red automática de monitoreo atmosférico. 
tas al ambiente de la ZMVT durante seis meses, de julio de 2016 a enero de 2017. Una vez transcurrido el periodo de exposición se retiraron y se trasladaron al laboratorio para su análisis.

\section{Preparación de muestras}

Una vez en el laboratorio, las bolsas con musgo se abrieron cuidadosamente para obtener el musgo expuesto (aproximadamente $2.5 \mathrm{~g}$ en peso seco por cada una). Posteriormente las muestras se molieron en un molino criogénico marca SPEX modelo 6770 en las siguientes condiciones: $5 \mathrm{~min}$ de preenfriamiento, 5 min de tiempo de molienda, y velocidad de 10 revoluciones por segundo.

Cabe mencionar que las muestras testigo (preexposición) no se almacenaron, sino que se analizaron al mismo tiempo que las bolsas con musgo fueron llevadas a los sitios de exposición y se prepararon y analizaron de la misma manera.

\section{Metales \\ $500 \mathrm{mg}$ de musgo (previamente molido) se co- locaron en vasos digestores y se agregaron $6 \mathrm{~mL}$ de $\mathrm{HNO}_{3}$ y $2 \mathrm{~mL}$ de $\mathrm{HCl}$. La digestión se llevó a cabo en un horno de microondas marca CEM modelo Mars 6 en las siguientes condiciones: rampa de tem- peratura: $20 \mathrm{~min}$; temperatura: $200^{\circ} \mathrm{C}$; presión: 180 psi; tiempo de mantenimiento: $15 \mathrm{~min}$, y potencia máxima: $1200 \mathrm{~W}$. Posteriormente, se adicionaron $2 \mathrm{~mL}$ de $\mathrm{H}_{2} \mathrm{O}_{2}$ y se colocaron nuevamente en el horno de microondas con un tiempo de mantenimiento de 20 min y temperatura $200{ }^{\circ} \mathrm{C}$ (CEM 2001, Poblano 2013). La muestra digerida se llevó a un volumen final de $10 \mathrm{~mL}$. La determinación de la concentración de metales se realizó por el método de adición de estándar interno, para lo cual se adicionaron $100 \mu \mathrm{L}$ de solución de Ge $(20 \mathrm{mg} / \mathrm{L})$ a una alícuota de $2 \mathrm{~mL}$ como estándar interno, y se homogeneizaron durante un minuto en un vortex (IAEA 1997). Por último, para analizar la muestra, $10 \mu \mathrm{L}$ de la muestra se colocaron en un reflector de cuarzo y se dejaron secar bajo luz infrarroja (DIN 2001).}

\section{Glutatión y fitoquelatinas}

Se colocaron $150 \mathrm{mg}$ de musgo molido criogénicamente en viales con espacio superior vacío (headspace), se taparon y la atmósfera de aire se sustituyó por gas nitrógeno de alta pureza. Posteriormente se adicionaron $4 \mathrm{~mL}$ de una solución de $200 \mathrm{mM}$ de DL-ditiotreitol bioextra $\geq 99.0 \%\left(\leq 4{ }^{\circ} \mathrm{C}\right)$ como estabilizante y se la mezcla se agitó durante $10 \mathrm{~min}$ a $3000 \mathrm{rpm}$ en un vórtex. Una alícuota de $1 \mathrm{~mL}$ se filtró a través de un filtro de nylon de $0.2 \mu \mathrm{m}$, la cual se transfirió a viales para cromatografía de $2 \mathrm{~mL}$ para su análisis (Bardarov et al. 2015).

\section{Análisis de muestras \\ Determinación de metales por fluorescencia de rayos $X$ en reflexión total}

La concentración de $\mathrm{Cu}, \mathrm{Zn}$, y $\mathrm{Pb}$ se determinó mediante la técnica de fluorescencia de rayos $\mathrm{X}$ en la modalidad de reflexión total (TXRF, por sus siglas en inglés), para lo cual se utilizó un espectrómetro de fluorescencia de rayos $\mathrm{X}$ marca Bruker modelo S2 Picofox con tubo de Mo $(50 \mathrm{kV}$ y $600 \mu \mathrm{A}) \mathrm{y}$ detector de Si-Li Flash ${ }^{\circledR}$ Drift, con una resolución $<$ $149 \mathrm{eV}$ para Mn K. Todas las muestras se analizaron por triplicado durante $300 \mathrm{~s}$ (Zarazúa-Ortega et al. 2013). La concentración de $\mathrm{Cu}, \mathrm{Zn}$ y $\mathrm{Pb}$ se obtuvo por el método de sensibilidades teóricas con estándar interno aplicando el programa Spectra Picofox (DIN 2001).

Como parte del control de calidad de los resultados se evaluó la exactitud y precisión del método, se procesaron y analizaron por triplicado dos materiales de referencia (NBS 1573 Tomatoe Leaves y IAEA Plant PTXRF-IAEA12) y seis blancos de reactivos en dos días consecutivos y una tercera vez durante el análisis de las muestras. Para evaluar la tasa de enriquecimiento de $\mathrm{Cu}, \mathrm{Zn}$ y $\mathrm{Pb}$ en los musgos, éstos se analizaron antes (concentración preexposición) y después (concentración final) del periodo de exposición.

\section{Determinación de glutatión y fitoquelatinas por cromatografía de líquidos}

Además de la concentración de GSH se determinó la concentración de fitoquelatinas PC2, PC3 y PC4 como posible mecanismo de homeostasis de los metales en estudio, dado que previamente se ha reportado el aumento de la producción de éstas (Petraglia et al. 2014). El análisis se realizó por triplicado empleando la técnica de cromatografía de líquidos de ultra alta resolución acoplada a espectrometría de masas (UPLC-MS por sus siglas en inglés) con un equipo marca Waters modelo Acquity Clase H. El método de análisis fue en fase reversa empleando una columna cromatográfica Poroshell 120 EC-C18 $2.7 \mu \mathrm{m} 100$ $\AA 3 \times 150 \mathrm{~mm}^{2}$ a $10{ }^{\circ} \mathrm{C}$ y como fase móvil A una mezcla de agua tipo I con acetonitrilo grado masas $>99.9 \%$ en proporción $95-5 \%$. La fase móvil B fue una mezcla de acetonitrilo grado masas $>99.9 \%$ con agua tipo I en proporción 90-10 \%. Ambas fases se acidificaron con ácido fórmico grado masas $>98 \%$ al $0.1 \%$. El tiempo de análisis fue de 10 min en modo gradiente a un flujo constante de $0.45 \mathrm{~mL} / \mathrm{min}$, iniciando en $100 \%$ A con cambio de proporción a $80 \%$ 
$\mathrm{A} / 20 \% \mathrm{~B}$ de 0 a $5 \mathrm{~min}$, de 5 a 6 min cambio de $80 \% \mathrm{~A} / 20 \% \mathrm{~B}$ a $100 \%$ de $\mathrm{B}$, de 6 a 8 min se mantuvo en $100 \%$ de B, de 8 a 8.5 min se cambió de $100 \%$ B a $100 \%$ A y finalmente de 8 a 10 min se mantuvo en $100 \%$ A. El tipo de ionización fue electroespray en modo positivo (ESI+), con voltaje de cono de $15 \mathrm{~V}$, voltaje capilar de $1.5 \mathrm{~K}$ y lecturas de masas en un rango de 200 a 1250 UMA. El volumen de inyección fue de $10 \mu \mathrm{L}$ para cada muestra (Bardarov et al. 2015). Para determinar la concentración de GHS y PC se prepararon curvas de calibración en concentraciones de 0.01 a $10 \mathrm{mg} / \mathrm{L}$ empleando estándares de referencia de GSH, así como de PC2, PC3 y PC4 con pureza $>95 \%$ marca AnaSpec.

\section{Análisis de materiales de referencia}

Con el objeto de evaluar la exactitud y precisión de los resultados, se analizaron en las mismas condiciones que las muestras dos materiales de referencia: NBS 1573 Tomatoe Leaves y IAEA Plant PTXRF-IAEA12. Se determinaron el coeficiente de variación $(\mathrm{CV})$, el porcentaje de recuperación ( $\mathrm{R} \%)$ y porcentaje de error calculado como:

Concentración medida - concentración certificada/concentración certificada $\times 100$

\section{Análisis estadístico}

Los datos se procesaron con el software IBM SPSS Statistics v. 26.0 (IBM 2019). Se emplearon pruebas no paramétricas para el procesamiento de datos debido al bajo número de muestras por sitio $(\mathrm{n}=2)$ (Moses 1952). Se realizó la prueba de rangos con signo de Wilcoxon para determinar si había diferencias significativas ente las concentraciones preexposición y postexposición. Para determinar las diferencias estadísticamente significativas entre los sitios de monitoreo se utilizó la prueba de Kruskal-
Wallis, al tiempo que se realizaron comparaciones post hoc por pares de sitios empleando la prueba $U$ de Mann-Whitney corregida por Bonferroni. También se realizaron correlaciones por rango de Spearman para estimar si había correlación entre las concentraciones de $\mathrm{Cu}, \mathrm{Zn}$ y $\mathrm{Pb}$ con la del GSH (ambos en $\mathrm{nM} / \mathrm{g}$ ). Todas las pruebas estadísticas se realizaron a un nivel de confianza del $95 \%$. Dado que en el análisis cromatográfico las concentraciones de PC2, PC3 y PC4 se encontraron por debajo del límite de detección no se realizaron pruebas estadísticas.

\section{RESULTADOS Y DISCUSIÓN}

Las concentraciones de $\mathrm{Cu}, \mathrm{Zn}$ y $\mathrm{Pb}$ obtenidas durante el análisis para los materiales de referencia NBS 1573 Tomatoe Leaves e IAEA Plant PTXRFIAEA12 mostraron la confiabilidad de los resultados obtenidos en este trabajo. Se obtuvieron coeficientes de variación menores al $10 \%$ que demostraron la precisión del método empleado, así como porcentajes de recuperación de 94 a $105 \%$ y errores menores al $5 \%$ que demostraron la se exactitud (Cuadro II).

Los musgos contienen $\mathrm{Cu}$ y $\mathrm{Zn}$, ya que éstos son elementos esenciales presentes en diversas proteínas y enzimas implicadas en funciones metabólicas como la activación de sistemas enzimáticos, regulación de la expresión genética y reacciones de oxidaciónreducción; sin embargo, en concentraciones mayores pueden causar daño, al igual que elementos considerados no esenciales como el $\mathrm{Pb}$, que no desempeña una función biológica conocida en plantas y es considerado como elemento tóxico (Navarro-Aviño et al. 2007).

Considerando lo anterior se evaluó la concentración de metales en estudio antes y después de la exposición de los musgos. Como se observa en los

CUADRO II. COMPARACIÓN ENTRE LA CONCENTRACIÓN MEDIDA Y LA CONCENTRACIÓN CERTIFICADA ( \pm DESVIACIÓN ESTÁNDAR) DE LOS MATERIALES DE REFERENCIA.

\begin{tabular}{|c|c|c|c|c|c|c|c|}
\hline MR & Elemento & $\begin{array}{l}\text { Concentración } \\
\text { medida }(\mathrm{mg} / \mathrm{kg})\end{array}$ & $\begin{array}{c}\text { Concentración } \\
\text { certificada }(\mathrm{mg} / \mathrm{kg})\end{array}$ & $\begin{array}{c}\mathrm{LD} \\
(\mathrm{mg} / \mathrm{kg})\end{array}$ & $\begin{array}{l}\text { CV } \\
(\%)\end{array}$ & $\begin{array}{l}\mathrm{R} \\
(\%)\end{array}$ & $\begin{array}{c}\text { Error } \\
(\%)\end{array}$ \\
\hline \multirow{3}{*}{$\begin{array}{l}\text { Tomato Leaves } \\
\text { NBS } 1573\end{array}$} & $\mathrm{Cu}$ & $11.50 \pm 0.28$ & \pm 1.0 & 0.014 & 2.4 & 104.5 & +4.5 \\
\hline & $\mathrm{Zn}$ & $62.19 \pm 0.88$ & $62 \pm 6.0$ & 0.013 & 1.4 & 100.3 & +0.3 \\
\hline & $\mathrm{Pb}$ & $6.59 \pm 0.25$ & $6.3 \pm 0.3$ & 0.020 & 3.8 & 104.7 & +4.7 \\
\hline \multirow{3}{*}{$\begin{array}{l}\text { Plant PTXRF- } \\
\text { IAEA12 }\end{array}$} & $\mathrm{Cu}$ & $9.73 \pm 0.52$ & $10.3 \pm 0.6$ & 0.014 & 5.3 & 94.5 & -5.5 \\
\hline & $\mathrm{Zn}$ & $104.36 \pm 0.54$ & $107 \pm 4.0$ & 0.013 & 0.5 & 97.5 & -2.5 \\
\hline & $\mathrm{Pb}$ & $6.45 \pm 0.40$ & $6.16 \pm 0.4$ & 0.020 & 6.2 & 104.7 & -4.7 \\
\hline
\end{tabular}

MR: material de referencia, LD: límite de detección, CV: coeficiente de variación, R: recuperación. 
resultados del cuadro III, y de acuerdo con el análisis estadístico de Wilcoxon $(p<0.05)$, hay diferencias entre la concentración previa y posterior al periodo de exposición, siendo esta última mayor. Esto permite demostrar que L. angustata es un buen acumulador de metales aerotransportados, lo cual es esencial en la técnica de monitoreo activo con bolsas con musgo (Ares et al. 2015, Vuković et al. 2015).

La concentración obtenida después del periodo de exposición - a excepción de los musgos de los sitios de Tollocan y San Mateo Atenco- muestra la siguiente tendencia: $\mathrm{Zn}>\mathrm{Cu}>\mathrm{Pb}$. Por otro lado, el orden decreciente de la concentración total de metales en los sitios de monitoreo fue: Tollocan $>$ Reforma $>$ San Mateo Atenco $>$ Alameda $>$ Aeropuerto $>$ San Cristóbal $>$ Metepec $>$ Ceboruco $>$ Toluca Centro $>$ Oxtotitlán. Aplicando las pruebas de Kruskal-Wallis y post hoc U de Mann-Whitney se determinaron diferencias estadísticas significativas $(p<0.05)$ entre sitios; en el caso del $\mathrm{Cu}$ se observaron diferencias entre Tollocan y Toluca Centro, Oxtotitlán, Ceboruco, Aeropuerto y San Cristóbal, y de este último con Alameda y Reforma. Con relación al Zn, las diferencias fueron entre Tollocan y Toluca Centro, Oxtotitlán, Ceboruco, Metepec y Reforma, y de este último con Toluca Centro. Finalmente, respecto del $\mathrm{Pb}$ se observaron diferencias entre Tollocan y Toluca Centro y San Cristóbal; entre San Mateo Atenco y Oxtotitlán, San Cristobal, Ceboruco, y Toluca Centro, y de éste con Alameda.

Los resultados obtenidos del biomonitoreo con bolsas con musgo pueden ser comparables con los obtenidos aplicando el monitoreo pasivo, ya que en general se presentó la misma tendencia; no obstante, con el primero fue posible calcular la tasa de enriquecimiento (concentración final/concentración preexposición) de $\mathrm{Cu}, \mathrm{Zn}$ y $\mathrm{Pb}$ para un determinado tiempo, que en este estudio fue de seis meses. A partir del análisis de los resultados que se muestran en el cuadro IV se pueden formar tres grupos: el primero

CUADRO III. CONCENTRACIÓN PRE Y POSEXPOSICIÓN ( \pm DESVIACIÓN ESTÁNDAR) DE Cu, Zn, Pb Y GLUTATIÓN EN Leskea angustata.

\begin{tabular}{|c|c|c|c|c|c|}
\hline & 1 - & $\mathrm{Cu}$ & $\mathrm{Zn}$ & $\mathrm{Pb}$ & GHS \\
\hline & g/Kg) & $(\mathrm{mg} / \mathrm{kg})$ & $(\mathrm{mg} / \mathrm{kg})$ & $(\mathrm{nM} / \mathrm{g})$ & \\
\hline & posición & 17.64 & 39.90 & 6.41 & 142.15 \\
\hline & San Mateo Atenco & $\begin{array}{r}142.85 \\
\pm 1.42\end{array}$ & $\begin{array}{r}252.94 \\
\pm 0.50\end{array}$ & $\begin{array}{l}196.72 \\
\pm 2.26\end{array}$ & $\begin{array}{r}458.80 \\
\pm 22.94\end{array}$ \\
\hline & Tollocan & $\begin{array}{r}212.28 \\
\pm 1.32\end{array}$ & $\begin{array}{r}537.95 \\
\pm 16.09\end{array}$ & $\begin{array}{l}169.92 \\
\pm 3.80\end{array}$ & $\begin{array}{r}808.29 \\
\pm 40.41\end{array}$ \\
\hline & Aeropuerto & $\begin{array}{r}127.52 \\
\pm 0.24\end{array}$ & $\begin{array}{r}307.89 \\
\pm 5.19\end{array}$ & $\begin{array}{r}24.48 \\
\pm 0.91\end{array}$ & $\begin{array}{r}381.48 \\
\pm 19.07\end{array}$ \\
\hline & San Cristóbal & $\begin{array}{l}118.55 \\
\pm 0.78\end{array}$ & $\begin{array}{r}230.56 \\
\pm 3.03\end{array}$ & $\begin{array}{r}18.61 \\
\pm 0.43\end{array}$ & $\begin{array}{r}283.89 \\
\pm 14.19\end{array}$ \\
\hline & Reforma & $\begin{array}{l}169.9 \\
\pm 2.24\end{array}$ & $\begin{array}{r}450.56 \\
\pm 2.11\end{array}$ & $\begin{array}{r}41.93 \\
\pm 1.28\end{array}$ & $\begin{array}{r}604.59 \\
\pm 30.23\end{array}$ \\
\hline Posexposicion & Alameda & $\begin{array}{r}161.75 \\
\pm 2.22\end{array}$ & $\begin{array}{r}306.61 \\
\pm 0.74\end{array}$ & $\begin{array}{r}53.28 \\
\pm 0.74\end{array}$ & $\begin{array}{r}455.30 \\
\pm 22.76\end{array}$ \\
\hline & Toluca Centro & $\begin{array}{l}112.46 \\
\pm 0.68\end{array}$ & $\begin{array}{l}195.08 \\
\pm 4.77\end{array}$ & $\begin{array}{r}17.48 \\
\pm 0.47\end{array}$ & $\begin{array}{r}296.71 \\
\pm 14.84\end{array}$ \\
\hline & Metepec & $\begin{array}{r}129.58 \\
\pm 0.60\end{array}$ & $\begin{array}{r}212.17 \\
\pm 9.66\end{array}$ & $\begin{array}{r}19.70 \\
\pm 0.26\end{array}$ & $\begin{array}{r}270.93 \\
\pm 10.55\end{array}$ \\
\hline & Ceboruco & $\begin{array}{r}126.32 \\
\pm 0.70\end{array}$ & $\begin{array}{r}194.28 \\
\pm 5.08\end{array}$ & $\begin{array}{r}18.63 \\
\pm 0.82\end{array}$ & $\begin{array}{r}282.36 \\
\pm 14.12\end{array}$ \\
\hline & Oxtotitlán & $\begin{array}{r}122.85 \\
\pm 1.59\end{array}$ & $\begin{array}{r}186.72 \\
\pm 3.19\end{array}$ & $\begin{array}{r}12.94 \\
\pm 1.18\end{array}$ & $\begin{array}{l}164.73 \\
\pm 3.24\end{array}$ \\
\hline
\end{tabular}


CUADRO IV. TASA DE ENRIQUECIMIENTO DE $\mathrm{Cu}, \mathrm{Zn}$ y $\mathrm{Pb}$ y PORCENTAJE DE INDUCCIÓN DE GLUTATIÓN EN Leskea angustata.

\begin{tabular}{|c|c|c|c|c|}
\hline \multirow[t]{2}{*}{ Sitio de Muestreo } & \multicolumn{3}{|c|}{$\begin{array}{l}\text { Tasa de enriquecimiento } \\
\qquad / 6 \text { meses }\end{array}$} & \multirow{2}{*}{$\begin{array}{c}\% \mathrm{DE} \\
\text { inducción }\end{array}$} \\
\hline & $\mathrm{Cu}$ & $\mathrm{Pb}$ & $\mathrm{Zn}$ & \\
\hline Tollocan & 12.03 & 26.51 & 13.48 & 569 \\
\hline San Mateo Atenco & 8.10 & 30.69 & 6.34 & 323 \\
\hline Reforma & 9.63 & 6.54 & 11.29 & 425 \\
\hline Alameda & 9.17 & 8.31 & 7.68 & 320 \\
\hline Aeropuerto & 7.23 & 3.82 & 7.72 & 268 \\
\hline San Cristóbal & 6.72 & 2.90 & 5.78 & 200 \\
\hline Metepec & 7.35 & 3.07 & 5.32 & 191 \\
\hline Ceboruco & 7.16 & 2.91 & 4.87 & 199 \\
\hline Toluca Centro & 6.38 & 2.73 & 4.89 & 209 \\
\hline Oxtotitlán & 6.96 & 2.02 & 4.68 & 116 \\
\hline
\end{tabular}

*Se obtuvieron correlaciones estadísticamente positivas $(\mathrm{r}>0.8)$ entre la concentración de $\mathrm{Cu}, \mathrm{Zn}$ y $\mathrm{Pb}$ y la inducción de GSH.

integrado por los musgos de Tollocan, San Mateo Atenco, Reforma y Alameda, que presentan las tasas de enriquecimiento más altas; el segundo constituido por los musgos de Aeropuerto, San Cristóbal y Metepec; y el tercero, con las tasas más bajas, compuesto por los muesgos de Ceboruco Toluca Centro y Oxtotitlán. De esta manera se puede observar cuáles son los sitios más y menos contaminados. Además de las diferencias en el nivel de exposición a metales pesados, las condiciones climáticas locales, las características físicas y químicas de los elementos, y la cantidad y tipo de sedimentación atmosférica podrían explicar parte de las diferencias en las concentraciones de metales entre sitios de muestreo (Giordano et al. 2013, Gerdol et al. 2014, Salo y Mäkinen 2014, Fernández et al. 2015, Varela et al. 2015).

En la ZMVT, las concentraciones de MP se deben principalmente a emisiones vehiculares e industriales, la resuspensión de suelos erosionados y zonas agrícolas, el polvo de vialidades sin pavimentar y las actividades de la construcción (SMA 2012). Particularmente el $\mathrm{Cu}$, el $\mathrm{Zn}$ y el $\mathrm{Pb}$ pueden ser emitidos por fuentes antrópicas asociadas a las industrias automotriz, del vidrio, metal-mecánica y química; también pueden deberse a emisiones de vehículos provenientes del desgaste de frenos y la abrasión de neumáticos. Los compuestos de $\mathrm{Zn}$ se utilizan como aditivos antidesgaste para el aceite de motor, por lo que las emisiones de gases del escape son una fuente importante en la contribución de partículas de este mineral (Thorpe y Harrison 2008, Kam et al. 2013, Świetlik et al. 2015).
De acuerdo con lo descrito anteriormente, la presencia de $\mathrm{Pb}, \mathrm{Cu}$ y $\mathrm{Zn}$ en el MP de los sitios Ceboruco y Metepec se asocia principalmente con la emisión de gases generados por ladrilleras y vehículos automotores; pero al localizarse al sur de la ZMVT, la dirección de los vientos propicia la dispersión de contaminantes (Fig. 1). En los sitios de Tollocan y Aeropuerto, ubicados en la zona industrial Toluca-Lerma, el MP proviene probablemente de las industrias del cromo, automotriz y metalmecánica, así como de procesos de combustión (Zarazúa-Ortega et al. 2011, 2013, SMA 2012). Además, al igual que San Cristóbal, San Mateo Atenco, Toluca Centro, Oxtotitlán, Alameda y Reforma, estas zonas presentan un intenso tráfico vehicular, por lo que éste se considera como la principal fuente de elementos de estudio en el MP del aire (Aničić et al. 2007, Barandovski et al. 2015, Pan et al. 2015, Świetlik et al. 2015). No onbstante, Toluca Centro y Oxtotitlán, son los sitios que presentaron la menor concentración de metales, lo cual puede deberse a que la estación de monitoreo de Toluca Centro está rodeada por árboles de gran altura y Oxtotitlán se encuentra el este del cerro de Coatepec, donde se encuentra la cuidad universitaria, lo cual puede actuar como barrera (SMA 2012).

Respecto a los sitios Alameda y Reforma, el tráfico vehicular se presenta sobre vialidades primarias; sin embargo, es importante mencionar que están rodeadas por edificios, lo cual puede contribuir a la existencia de una obstrucción a la dispersión de partículas de $\mathrm{Pb}, \mathrm{Cu}$ y $\mathrm{Zn}$ asociados al MP, incluyendo las provenientes de industrias metalmecánicas y cromadoras ubicadas en la zona industrial de Toluca. Lo anterior concuerda con lo reportado por ZarazúaOrtega et al. (2013), Caballero-Segura et al. (2014) y Macedo-Miranda et al. (2016), quienes en estudios previos encontraron briofitas altamente enriquecidas por estos metales $(\mathrm{FE} \geq 10)$ en dichos sitios, aplicando monitoreo pasivo.

Por otro lado, a diferencia de las plantas superiores, en musgos, el GHS participa en una gran variedad de procesos y puede desempeñar un papel esencial en la destoxificación por metales divalentes, la cual se caracteriza por un aumento del grupo de GSH con o sin evidencia de participación de PC (Bruns et al. 2001, Saxena et al. 2003, Bleuel et al. 2005, Braha et al. 2007, Rau et al. 2007, Leinenweber et al. 2009). En este estudio se observó un aumento estadístico significativo en la concentración de GHS (Cuadro III); sin embargo, la concentración de PC fue menor al límite de detección $(0.2 \mathrm{nM} / \mathrm{g})$.

En este sentido, como se observa en la figura 2, la concentración de GHS siguió casi la misma 

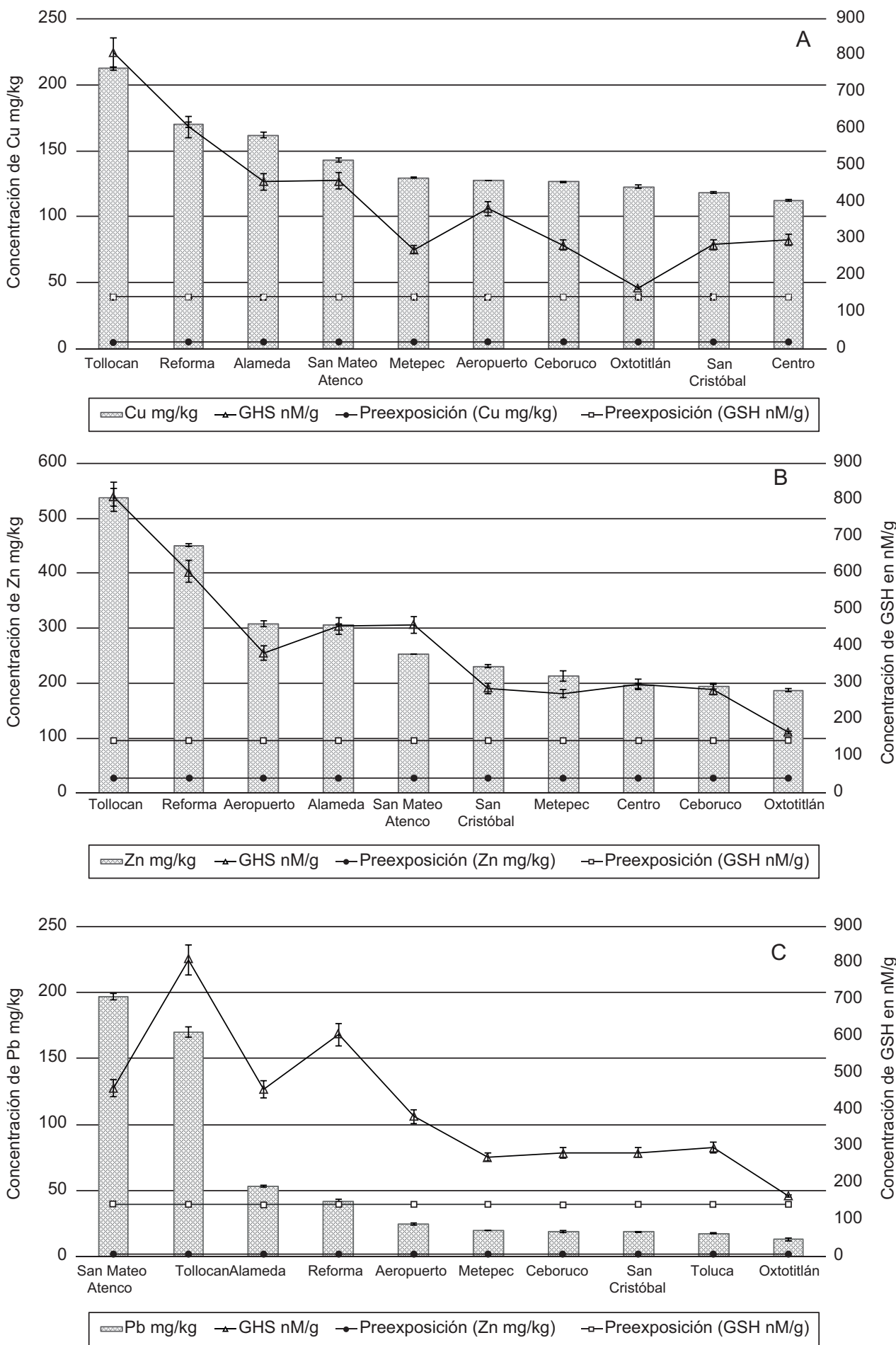

Fig. 2. Concentración pre y posexposición de (a) $\mathrm{Cu}$, (b) $\mathrm{Zn}$ y (c) $\mathrm{Pb}$ (los tres en $\mathrm{mg} / \mathrm{kg}$ ) vs. glutatión (GHS, en $\mathrm{nM} / \mathrm{g}$ ) en Leskea angustata, en los sitios de monitoreo de la zona metropolitana del valle de Toluca. 
tendencia que la de $\mathrm{Cu}, \mathrm{Zn}$ y $\mathrm{Pb}$ en los musgos para cada sitio de muestreo. Para el sitio de Tollocan, que presentó la mayor acumulación de metales en estudio, las briofitas presentaron un aumento del $569 \%$ en la producción de GHS, y en las ubicadas en Oxtotitlán este incremento fue del $116 \%$ respecto de la concentración preexposición (Cuadro IV). Estos resultados concuerdan con lo reportado por Varela et al. (2018), quienes encontraron mayor concentración de GHS en los musgos más cercanos a las fábricas, los cuales a su vez presentaron altas concentraciones de $\mathrm{Cd}, \mathrm{Pb}$ y Ni.

Finalmente, para estimar si existe correlación entre la concentración de $\mathrm{Cu}, \mathrm{Zn}$ y $\mathrm{Pb}$ y la concentración-inducción de GSH, se realizaron correlaciones de Spearman entre cada metal y el GSH. Los resultados mostraron las siguientes correlaciones positivas: GSH-Cu: $\mathrm{r}=0.8$; GSH-Zn: $r=0.9$, y GSH-Pb: $r=$ 0.9 . Por lo tanto, se infiere que las concentraciones de los metales en estudio tienen relación directa con la inducción de GSH.

Esto es indicativo de que, en presencia de altos niveles de metales, el GHS puede quelarlos y transformarlos en nuevos compuestos menos tóxicos con diferentes propiedades químicas, evitando así el daño celular. Así, este compuesto antioxidante permitiría que el $L$. angustata mantenga una presencia abundante en la ZMVT (Zepeda-Gómez et al. 2012) en sitios altamente contaminados por metales, y pueda ser utilizado para el biomonitoreo de contaminación atmosférica por metales (Saxena y Saxena 2012, Varela et al. 2018).

\section{CONCLUSIONES}

La presencia de $\mathrm{Cu}, \mathrm{Zn}$ y $\mathrm{Pb}$ en musgos expuestos en la ZMVT indica un origen principalmente antrópico asociado tanto a fuentes móviles (automotores) como a la industria asentada en esa zona.

El biomonitoreo activo con bolsas con musgo es una técnica que provee información importante acerca de la contaminación atmosférica por metales aerotransportados, ya que es posible calcular la tasa de enriquecimiento de los contaminantes en un tiempo de exposición determinado.

El musgo Leskea angustata es un organismo con características adecuadas (es endémico, buen acumulador y presenta alta tolerancia a metales) para utilizarse en estudios de contaminación atmosférica por metales.

Aparentemente, las PC en Leskea angustata de la ZMVT no desempeñan un papel importante en la destoxificación de metales.
El GHS desempeña un papel fundamental en la bioacumulación de metales divalentes en Leskea angustata de la ZMVT aumentando el potencial de acumulación de metales, ya que es un mecanismo importante de detoxificación en estos organismos.

\section{REFERENCIAS}

Adamo P., Giordano S., Vingiani S., Cobianchi C. y Violante P. (2003). Trace element accumulation by moss and lichen exposed in bags in the city of Naples (Italy). Environ. Pollut. 122, 91-103. https://doi.org/10.1016/ S0269-7491(02)00277-4

Aničić M., Frontasyeva M.V., Tomašević M. y Popović A. (2007). Assessment of atmospheric deposition of heavy metals and other elements in Belgrade using the moss biomonitoring technique and neutron activation analysis. Environ. Monit. Assess. 129, 207-219. https://doi.org/https://doi.org/10.1007/ s10661-006-9354-y

Aničić M., Tasić M., Frontasyeva V., Tomašević M., Rajšić S., Mijić Z. y Popović A. (2009). Active moss biomonitoring of trace elements with Sphagnum girgensohnii moss bags in relation to atmospheric bulk deposition in Belgrade, Serbia. Environ. Pollut. 157 (2), 673-679. https://doi.org/10.1016/j.envpol.2008.08.003

Ares A., Aboal J.R., Carballeira A., Giordano S., Adamo P. y Fernández J.A. (2012). Moss bag biomonitoring: A methodological review. Sci. Total Environ. 432, 14358. https://doi.org/10.1016/j.scitotenv.2012.05.087

Ares A., Aboal J., Carballeira A. y Fernández J.A. (2015). Do moss bags containing devitalized Sphagnum denticulatum reflect heavy metal concentrations in bulk deposition? Ecological Indicators 50, 90-98. https:// doi.org/10.1016/j.ecolind.2014.10.030

Bačeva K., Stafilov T., Šajn R. y Tănăselia C. (2013). Air dispersion of heavy metals in the vicinity of the As$\mathrm{Sb}-\mathrm{Tl}$ abounded mine and responsiveness of moss as a biomonitoring media in small-scale investigations. Environ. Sci. Pollut. Res. 20 (12), 8763-8779. https:// doi.org/10.1007/s11356-013-1845-0

Barandovski L., Frontasyeva M.V., Stafilov T., Šajn R. y Ostrovnaya T.M. (2015). Multi-element atmospheric deposition in Macedonia studied by the moss biomonitoring technique. Environ. Sci. Pollut. Res. 22 (20), 16077-16097. https://doi.org/10.1007/s11356015-4787-x

Bardarov K., Naydenov M. y Djingova R. (2015). HPLCHRMS method for fast phytochelatins determination in plants. Application to analysis of Clinopodium vulgare L. Talanta 142, 20-27. https://doi.org/10.1016/j. talanta.2015.04.014 
Basile A., Sorbo S., Pisani T., Paoli L., Munzi S. y Loppi S. (2012). Bioacumulation and ultrastructural effects of $\mathrm{Cd}, \mathrm{Cu}, \mathrm{Pb}$ and $\mathrm{Zn}$ in the moss Scorpiurum circinatum (Brid.) Fleisch. \& Loeske. Environ. Pollut. 166, 208-211. https://doi.org/10.1016/j.envpol.2012.03.018

Bleuel C., Wesenberg, D., Sutter K., Miersch J., Braha B., Bärlocher, F. y Krauss G.J. (2005). The use of the aquatic moss Fontinalis antipyretica L. ex Hedw. as a bioindicator for heavy metals: $3 . \mathrm{Cd}^{2+}$ accumulation capacities and biochemical stress response of two Fontinalis species. Sci. Total Environ. 345 (1), 13-21. https://doi.org/10.1016/j. scitotenv.2004.11.015

Braha B., Tintemann H., Krauss G., Ehrman J., Bärlocher F. y Krauss G.J. (2007). Stress response in two strains of the aquatic hyphomycete Heliscus lugdunensis after exposure to cadmium and copper ions. Biometals 20 (1), 93-105. https://doi.org/10.1007/s10534-0069018-y

Bruns I., Friese K., Markert B. y Krauss G.J. (1997). The use of Fontinalis antipyretica L. ex Hedw. as a bioindicator for heavy metals. 2. Heavy metal accumulation and physiological reaction of Fontinalis antipyretica L. ex Hedw. in active biomonitoring in the River Elbe. Sci. Total Environ. 204 (2), 161-176. https://doi. org/10.1016/S0048-9697(97)00174-5

Bruns I., Sutter K., Menge S., Neumann D. y Krauss G. (2001). Cadmium lets increase the glutathione pool in bryophytes. J. Plant Physiol. 158, 79-89. https://doi. org/10.1078/0176-1617-00071

Caballero-Segura B., Ávila-Pérez P., Barrera C.E., Ramírez J.J., Zarazúa G., Soria R. y Ortiz-Oliveros H.B. (2014). Metal content in mosses from the Metropolitan Area of the Toluca Valley: A comparative study between inductively coupled plasma optical emission spectrometry (ICP-OES) and total reflection X-ray fluorescence spectrometry (TXRF). Intern. J. Environ. Anal. Chem. 94 (13), 1288-1301. https://doi.org/10.1 080/03067319.2014.940343

Calabrese S., D’Alessandro W., Bellomo S., Brusca L., Martin R.S., Saiano F. y Parello F. (2015). Characterization of the Etna volcanic emissions through an active biomonitoring technique (moss-bags): Part 1 Major and trace element composition. Chemosphere 119, 1447-1455. https://doi.org/10.1016/j.chemosphere.2014.08.086

CEM (2001). Methods for microwave accelerated reaction system for acid digestion. CEM Corporation, Matthews, Carolina del Norte, EUA.

Clemens S. y Peršoh D. (2009). Multi-tasking phytochelatin synthases. Plant Sci. 177 (4), 266-271. https://doi. org/10.1016/j.plantsci.2009.06.008
Cobbett C. (2000). Phytochelatin biosynthesis and function in heavy-metal detoxification. Curr. Opin. Plant Biol. 3, 211-216. https://doi.org/10.1016/S13695266(00)80067-9

Cobbett C. y Goldsbrough P. (2002). Phytochelatins and metallothioneins: Roles in heavy metal detoxification and homeostasis. Annu. Rev. Plant Biol. 53, 159-182. https://doi.org/10.1146/annurev. arplant.53.100301.135154

Cocozza C., Ravera S., Cherubini P., Lombardi F., Marchetti M. y Tognetti R. (2016). Integrated biomonitoring of airborne pollutants over space and time using tree rings, bark, leaves and epiphytic lichens. Urban For. Urban Greening 17, 177-191. https://doi. org/10.1016/j.ufug.2016.04.008

Díaz C., Khalidou M., Iturbe A., Esteller M. y Reyna F. (2005). Características fisiográficas de una cuenca. En: Recursos hídricos: conceptos básicos y estudios de caso en Iberoamérica (Díaz-Delgado C., Esteller M., y López-Vera F., Eds.). Piriguazú, Toluca, México, pp. 5-17.

DIN (2001). EDIN 51003:2001-01: Total reflection x-ray fluorescence analysis (TXRF). Deutsches Institut für Normung EV, Alemania.

Dotor J. (2014). Evaluación de los metales $\mathrm{Cr}, \mathrm{Cu}, \mathrm{Zn}$, $\mathrm{Pb}$, y $\mathrm{Mn}$ en la atmósfera de la Zona Metropolitana del Valle de Toluca usando como indicador el depósito seco sobre hojas de trueno común (Ligutrum vulgare) y estimación del riesgo para la salud de la población expuesta. Tesis de Maestría. Facultad de Química, Universidad Autónoma del Estado de México, Toluca, Estado de México, México, 133 pp.

Emamverdian A., Ding Y., Mokhberdoran F. y Xie Y. (2015). Heavy metal stress and some mechanisms of plant defense response. Sci. World J. 2015. https://doi. org $/ 10.1155 / 2015 / 756120$

Fernández J.A., Boquete M.T., Carballeira A. y Aboal J.R. (2015). A critical review of protocols for moss biomonitoring of atmospheric deposition: sampling and sample preparation. Sci. Total Environ. 517, 132150. https://doi.org/10.1016/j.scitotenv.2015.02.050

Gekeler W., Grill E., Winnacker L. y Zenk MH (1989). Survey of the plant kingdom for the ability to bind heavy metals through phytochelatins. Z Naturforsch 44, 361-369. https://doi.org/10.1515/znc-1989-5-605

GEM (2014). Estaciones de monitoreo. Gobierno del Estado de México [en línea]. http://rama.edomex.gob. mx. 26/01/2017

Gerdol R., Marchesini R., Iacumin P. y Brancaleoni L. (2014). Monitoring temporal trends of air pollution in an urban area using mosses and lichens as biomonitors. Chemosphere 108, 388-395. https://doi.org/10.1016/j. chemosphere.2014.02.035 
Gil P. (2015). Biomarcadores de exposición a metales pesados en musgo de la ZMVT. Tesis de Maestría en Ciencias en Ingeniería Ambiental, Instituto Tecnológico de Toluca. Metepec, Estado de México, México, 142 pp.

Giordano S., Adamo P., Monaci F., Pittao E., Tretiach M. y Bargagli R. (2009). Bags with oven-dried moss for the active monitoring of airborne trace elements in urban areas. Environ. Pollut. 157 (10), 2798-2805. https:// doi.org/10.1016/j.envpol.2009.04.020

Giordano S., Adamo P., Spagnuolo V., Tretiach M. y Bargagli R. (2013). Accumulation of airborne trace elements in mosses, lichens and synthetic materials exposed at urban monitoring stations: Towards a harmonisation of the moss-bag technique. Chemosphere 90 (2), 292-99. https://doi.org/10.1016/j.chemosphere.2012.07.006

Gómez H.D., del Valle, R., Galarraga F., Hernández J., Roschman-González A. y Escalona A. (2013). Biomonitoreo activo de hidrocarburos aromáticos policíclicos en el aire del Valle de Caracas, Venezuela, empleando el liquen Parmotrema sancti-angelii (Lynge) Hale. Rev. Int. Contam. Ambient. 29, 261-67.

González A.G., Pokrovsky O.S., Beike A.K., Reski R., di Palma A., Adamo P., Giordano S. y Fernández J.A. (2016). Metal and proton adsorption capacities of natural and cloned Sphagnum mosses. J. Colloid Interf. Sci. 461, 326-334. https://doi.org/10.1016/j.jcis.2015.09.012

Grill E., Winnacker E. y Zenk M.H. (1991). Phytochelatins. Methods Enzymol. 205, 333-341. https://doi. org/10.1016/0076-6879(91)05115-C

IAEA (1997). Sampling, storage and sample preparation procedures for X-ray fluorescence analysis of environmental materials. Documento técnico. International Atomic Energy Agency, Viena, Austria, 55 pp.

IBM (2019). IBM SPSS Statistics para Windows, version 26.0. Corporación IBM. Armonk, Nueva York.

Kam W., Delfino R.J., Schauer J.J. y Sioutas C. (2013). A comparative assessment of $\mathrm{PM}_{2.5}$ exposures in lightrail, subway, freeway, and surface street environments in Los Angeles and estimated lung cancer risk. Environ. Sci. Processes Impacts 15 (1), 234-243. https://doi. org/10.1039/C2EM30495C

Kneer R. y Zenk M. (1992). Phytochelatins protect plant enzymes from heavy metal poisoning. Phytochemirstry 31 (8), 2663-2667. https://doi.org/10.1016/00319422(92)83607-Z

Keshavarzi B., Tazarvi Z., Rajabzadeh M.A. y Najmeddin A. (2015). Chemical speciation, human health risk assessment and pollution level of selected heavy metals in urban street dust of Shiraz, Iran. Atmos. Environ. 119, 1-10. https://doi.org/10.1016/j.atmosenv.2015.08.001

Konno H., Nakashima S. y Katoh K. (2010). Metaltolerant moss Scopelophila cataractae accumulates copper in the cell wall pectin of the protonema. J. Plant
Physiol. 167 (5), 358-364. https://doi.org/10.1016/j. jplph.2009.09.011

Krauss G. J., Krauss G., Menge S. y Wesenberg D. (2005). Cadmium stress response at the thiol peptide level in mosses and fungi. En: Sulfur transport and assimilation in plants in the post genomic era (Davidian J., Grill D., L. Kok de, Stulen I., Hawkesford M., Schnug E. y Rennenberg H., Eds.). Backhuys Publishers, Leiden, Países Bajos, 233-236.

Leinenweber, G., Stegen, S., y Díaz-Palma, P. (2009). Increase of total glutathione as a response to $\mathrm{Cd}$ induced stress in Chilean endemic bryophytes (Thuidium sp.). J. Chil. Chem. Soc. 54 (4), 401-404. https://doi. org/10.4067/S0717-97072009000400017

Macedo-Miranda G., Ávila-Pérez P., Gil-Vargas P., Zarazúa G., Sánchez-Meza J.C., Zepeda-Gómez C. y Tejeda S. (2016). Accumulation of heavy metals in mosses: A biomonitoring study. Springer Plus 5 (1), 715. https:// doi.org/10.1186/s40064-016-2524-7

Moses L.E. (1952). Non-parametric statistics for psychological research. Psychological Bulletin 49 (2), 122143. https://doi.org/10.1037/h0056813

Navarro-Aviño J.P., Aguilar-Alonso I. y López-Moya J.R. (2007). Aspectos bioquímicos y genéticos de la tolerancia y acumulación de metales pesados en planta. Rev. Ecosis. 16 (2), 10-25.

OMS (2016). Calidad del aire y salud. Organización Mundial de la Salud, Ginebra, Suiza [en línea]. http://www. who.int/mediacentre/factsheets/fs313/es/ 18/07/2017.

Pan Y., Tian S., Li X., Sun Y., Li Y., Wentworth G.R., y Wang Y. (2015). Trace elements in particulate matter from metropolitan regions of northern China: Sources, concentrations and size distributions. Sci. Total Environ. 537, 9-22. https://doi.org/10.1016/j. scitotenv.2015.07.060

Petraglia A., de Benedictis M., Degola F., Pastore G., Calcagno M., Ruotolo R. y Sanità di Toppi L. (2014). The capability to synthesize phytochelatins and the presence of constitutive and functional phytochelatin synthases are ancestral (plesiomorphic) characters for basal land plants. J. Exp. Bot. 65 (4), 1153-1163. https://doi.org/10.1093/jxb/ert472

Poblano J. (2013). Uso de briofitas como indicadores atmosféricos de metales pesados en la Zona Metropolitana del Valle de Toluca. Tesis de Licenciatura. Facultad de Ciencias, Universidad Autónoma del Estado de México, Toluca, Estado de México, México, 103 pp.

Rau S., Miersch J., Neumann D., Weber E. y Krauss G.J. (2007). Biochemical responses of the aquatic moss Fontinalis antipyretica to $\mathrm{Cd}, \mathrm{Cu}, \mathrm{Pb}$ and $\mathrm{Zn}$ determined by chlorophyll fluorescence and protein levels. Environ. Exp. Bot. 59 (3), 299-306. https://doi. org/10.1016/j.envexpbot.2006.03.001 
Salo H. y Mäkinen J. (2014). Magnetic biomonitoring by moss bags for industry-derived air pollution in SW Finland. Atmos. Environ. 97, 19-27. https://oi. org/10.1016/j.atmosenv.2014.08.003

Saxena A., Saxena D.K. y Srivastava H.S. (2003). The influence of glutathione on physiological effects of lead and its accumulation in moss Sphagnum squarrosum. Water Air Soil Poll. 143, 351-361. https://doi. org/10.1023/A:102285680

Saxena A. y Saxena A. (2012). Bioaccumulation and glutathione-mediated detoxification of copper and cadmium in Sphagnum squarrosum Crome Samml. Environ. Monit. Assess. 184, 4097-4103. https://doi. org/10.1007/s10661-011-2246-9.

SEMARNAT (2012). Norma Oficial Mexicana NOM156-SEMARNAT-2012. Establecimiento y operación de sistemas de monitoreo de la calidad del aire. Secretaria de Medio Ambiente y Recursos Naturales. Diario Oficial de la Federación, 16 de julio.

SMA (2012). Programa para mejorar la calidad del aire del Valle de Toluca (2012-2017). Secretaría del Medio Ambiente, Gobierno del Estado de México, 220 pp. [en línea]. http://proaire.edomex.gob.mx/ sites/proaire.edomex.gob.mx/files/files/mis\%20pdf/ ProAire\%20Valle\%20de\%20Toluca\%202012-2017. pdf. 08/10/2018

Špirić Z., Vučković I., Stafilov T., Kušan V. y Frontasyeva M. (2013). Air pollution study in Croatia using moss biomonitoring and ICP-AES and AAS analytical techniques. Arch. Environ. Contam. Toxicol. 65, 33-46. https://doi.org/10.1007/s00244-013-9884-6

Steffens J.C. (1990). The heavy metal-binding peptides of plants. Annu. Rev. Plant Biol. 41 (1), 553-575. https:// doi.org/10.1146/annurev.pp.41.060190.003005

Świetlik R., Trojanowska M., Strzelecka M. y BochoJaniszewska A. (2015). Fractionation and mobility of $\mathrm{Cu}, \mathrm{Fe}, \mathrm{Mn}, \mathrm{Pb}$ and $\mathrm{Zn}$ in the road dust retained on noise barriers along expressway-A potential tool for determining the effects of driving conditions on speciation of emitted particulate metals. Environ. Pollut. 196, 404-413. https://doi.org/10.1016/j.envpol.2014.10.018

Tejeda S., Martínez T. y Zarazúa-Ortega G. (2015). Concentration of toxic elements in topsoils of the Miguel Hidalgo natural park in Mexico. International Conference on Modern Trends in Activation Analysus. Delft, Holanda, 23 al 28 de agosto.

Thorpe A. y Harrison R.M. (2008). Sources and properties of non-exhaust particulate matter from road traffic: a review. Sci. Total Environ. 400, 270-282. https://doi. org/10.1016/j.scitotenv.2008.06.007

Tretiach M., Adamo P., Bargagli R., Baruffo L., Carletti L., Crisafulli P. y Pittao E. (2007). Lichen and moss bags as monitoring devices in urban areas. Part I: Influence of exposure on sample vitality. Environ. Pollut. 146 (2), 380-391. https://doi.org/10.1016/j.envpol.2006.03.046

Tretiach M., Pittao E., Crisafulli P. y Adamo P. (2011). Influence of exposure sites on trace element enrichment in moss-bags and characterization of particles deposited on the biomonitor surface. Sci. Total Environ. 409 (4), 822-830. https://doi.org/10.1016/j. scitotenv.2010.10.026

Varela Z., Fernández J.A., Real C., Carballeira A. y Aboal J.R. (2015). Influence of the physicochemical characteristics of pollutants on their uptake in moss. Atmos. Environ. 102, 130-135. https://doi.org/10.1016/j. atmosenv.2014.11.061

Varela Z., Debén S., Saxena D.K., Aboal J.R. y Fernández J.Á. (2018). Levels of antioxidant compound glutathione in moss from industrial areas. Atmosphere 9, 284-291. https://doi.org/10.3390/atmos9070284

Vuković G., Urošević M.A., Razumenić I., Kuzmanoski M., Pergal M., Škrivanj S. y Popović A. (2014). Air quality in urban parking garages $\left(\mathrm{PM}_{10}\right.$, major and trace elements, PAHs): Instrumental measurements vs. active moss biomonitoring. Atmos. Environ. 85, 31-40. https://doi.org/10.1016/j.atmosenv.2013.11.053

Vuković G., Urošević M.A., Tomašević M., Samson R. y Popović A. (2015). Biomagnetic monitoring of urban air pollution using moss bags (Sphagnum girgensohnii). Ecol. Indic. 52, 40-47. https://doi.org/10.1016/j. ecolind.2014.11.018

Zarazúa-Ortega G., Vázquez-Preciado S., Poblano-Bata J., Tejeda-Vega S., Ávila-Pérez P., Pérez-del Prado J. y Astivia-Segura F.. 2011. Caracterización morfológica de briofitas en la Zona Metropólitana del Valle de Toluca por microscopia electrónica de barrido. Informe Técnico ITGC AMB/14/2011. Centro Nuclear "Dr. Nabor Carrillo Flores”, Ocoyoacac, Estado de México, México, 19 pp.

Zarazúa-Ortega G., Poblano-Bata J., Tejeda-Vega S., Ávila-Pérez P., Zepeda-Gómez C., Ortiz-Oliveros H. y Macedo-Miranda G. (2013). Assessment of spatial variability of heavy metals in Metropolitan Zone of Toluca Valley, Mexico, using the biomonitoring technique in mosses and TXRF analysis. Analysis. Sci. World J. https://doi.org/10.1155/2013/426492

Zechmeister H.G., Grodzinska K. y Szarek-Lukasazewska G. (2003). Bryophytes. En: Bioindicators \& Biomonitors (Market B.A., Breure A.M. y Zechmeister H.G., Eds.). Elsevier Science, Oxford, Inglaterra, pp. 329419.

Zepeda-Gómez C., Ávila-Pérez P., Díaz-García U., AlanísMartínez Y., Zarazúa-Ortega G. y Amaya-Chávez A. (2012). Diversity of epiphytic mosses in the metropolitan area of the Toluca Valley, Mexico. Rev. Mex. Biodivers. 85, 298-302. https://doi.org/10.7550/rmb.35456 